\title{
Prone Positioning of Patients with Cervical Spine Pathology
}

\author{
Sarah L. Boyle ${ }^{1}$ Zoe Unger ${ }^{1}$ Vinay Kulkarni² Eric M. Massicotte ${ }^{2} \quad$ Lashmi Venkatraghavan $^{1}$ \\ ${ }^{1}$ Department of Anesthesia and Pain Medicine, Toronto Western \\ Hospital, University of Toronto, Toronto, Ontario, Canada \\ 2Division of Neurosurgery, Department of Surgery, Toronto Western \\ Hospital, University of Toronto, Toronto, Ontario, Canada

\begin{abstract}
Address for correspondence Zoe Unger, MD, FRCPC, Department of Anesthesia and Pain medicine, Toronto Western Hospital, 399 Bathurst Street, Toronto, Ontario M5T 2S8,
\end{abstract} \\ Canada (e-mail: Zoe.Unger@uhn.ca).
}

J Neuroanaesthesiol Crit Care 2020;7:70-76

\begin{abstract}
Keywords

- awake prone positioning

- cervical spine

- logroll

- prone

- self-positioning

Patients with cervical trauma or degenerative disease often require surgical decompression and stabilization in the prone position and are at the risk of secondary neurological injury during this transfer. This review aims to explore the current literature on different methods of positioning patients prone and to identify the safest technique to achieve prone positioning in patients with an unstable cervical spine undergoing posterior cervical spine surgery. We searched the Embase, Medline, and Medline-in Process databases for literature in English related to prone positioning patients with cervical spine pathology undergoing spine surgery. Seventy-three citations were identified as relevant and reviewed in detail with 20 articles being identified as answering the clinical questions posed. Our literature review identified three methods of prone positioning patients with cervical pathology: logroll with manual in-line stabilization (MILS), rotating the patient on a specialized spinal table using a "sandwich and flip" technique, and awake prone positioning. Each of these methods has its own advantages and disadvantages. When comparing the degree of neck movement between positioning techniques, "sandwich and flip" rotation was associated with over $50 \%$ reduction in both flexion-extension and axial-lateral rotation as compared to logroll with MILS. Awake self-positioning of a patient is another alternative that allows for rapid neurological assessment after repositioning. A "sandwich and flip" is the safest way to turn a patient with cervical pathology into a prone position for surgery. For cooperative patients, who are physically capable, awake self-positioning is a good alternative.
\end{abstract}

\section{Introduction}

Up to $10 \%$ of patients with acute cervical spinal cord injury have a neurological deterioration after the initial injury. ${ }^{1-5}$ Prevention of secondary neurological injury and its associated morbidity is, therefore, of paramount importance. Patients with unstable cervical spine pathology either due to trauma or degenerative disease often require surgical decompression and stabilization through a posterior approach. Perioperatively, these patients are at the risk of secondary neurological injury to the spinal cord from hypotension, hypoxia, and movement of the neck. Airway manipulation is the most common cause of anesthesia-related medico-legal claims in cervical surgery followed by positioning-related injuries. ${ }^{6}$ However, the majority of the claims related to airway manipulation appear to be a result of expanding neck hematomas and not an intubation-related injury. Currently, there is considerable research on the safe management of the airway in patients with an unstable cervical spine ${ }^{7-11}$ but little on safe positioning of these patients.

Patients are at an increased risk of secondary neurological injury during prone positioning not only due to the amount of neck movement on positioning but also due to hemodynamic changes associated with general anesthesia. ${ }^{7}$ Currently, there is no standard of care for safe prone positioning of patients with cervical spine pathology. Techniques used vary with institutional practices as well as the preferences of the individual surgeons. The aim of this review is to explore
License terms

(ㅇ) (1) $\ominus \circledast$ 
the current literature on different methods of positioning patients prone and to identify the safest technique to achieve prone positioning in patients with an unstable cervical spine undergoing posterior cervical spine surgery. We determined the safety of each technique by its ability to avoid secondary neurological injury, pressure-related injuries, and injuries to assisting medical personnel.

\section{Methods}

We searched the Embase, Medline, and Medline-in Process databases for literature related to prone positioning in patients with cervical spine pathology undergoing spine surgery until December 8th, 2017. Keywords used included "prone," “logroll," “Jackson," “rotation," "manual," “neck," "cervical spine," "decompression," "microdecompression," "laminectomy," "discectomy," and "stabilization." Additional articles were included from the references of the selected literature. There was no restriction on the type of study but citations not in English were excluded from the results.

After removing duplicate citations, each citation was reviewed to see if it was relevant to answer the following three main clinical questions:

1. What are the different approaches for the safe positioning of patients who are at risk of secondary spinal cord injury into the prone position and what are the advantages and disadvantages of each approach?

2. Which approach produces the least movement in the cervical spine and hence lowest risk of a secondary neurological injury?

3. Are there differences in safe positioning between patients with traumatic cervical pathology and those with degenerative cervical spine? If so, what are those differences?

The full texts were then obtained for all relevant citations and the articles' contributions to answering the clinical questions were assessed. All articles deemed to have a section that was relevant have been included in this review article.

\section{Results}

The initial literature search identified 9,875 citations that are in the English language. After removing duplicates $(1,951)$, 75 citations were identified as having potential relevance and the full texts were reviewed. Fifty-five articles were excluded for not answering our clinical questions ( - Fig. 1 ).

Out of 20 articles that are included in the review, 6 articles had a section that described prone positioning techniques for patients with cervical pathology, 10 articles described methods of awake self-positioning of patients into the prone position, and 4 cadaveric studies compared cervical spine movement between rotating the patient using a Jackson table (Mizuho OSI; Union City, California United States), and manual inline stabilization (MILS). There were no studies specifically looking at the amount of neck movement in degenerative versus traumatic cervical pathology.

\section{Different approaches to prone positioning}

Three approaches to prone positioning patients with an unstable cervical spine were identified in the literature: $\log$ roll with MILS, "sandwich and flip" technique, and awake self-positioning of patients ( - Table $\mathbf{1}$ ).

\section{A. Logroll method with MILS}

The logroll method with MILS requires one person to keep the patient's head in line with the shoulders, while at least three other team members roll the patient from the supine to the prone position. ${ }^{12}$ Coordination and close communication between all of the team members are essential during the logroll to ensure that the spine remains inline at all times. The major advantage of MILS is that it requires no additional equipment, making the maneuver faster and more efficient to perform. The main limitation is the difficulty of achieving precise and coordinated movement by all members of the team to maintain the spine inline during positioning. ${ }^{12}$

\section{B. "Sandwich and flip" rotation}

There are many special spinal surgery tables (Jackson table [Mizuho OSI; Union City, California, United States], Allen's table [Allen Medical; Acton, Massachusetts, United States], and Galen spinal table [Southern Medical; Irene, Gauteng, South Africa]) that use the "sandwich and flip" technique. Each of these tables consists of two parts-a flat board for placing the patient supine and a carbon frame with prone supports for the chest and hips that is used for patients in the prone position (-Figs. 2 and 3). These tables have a built-in rotation mechanism that allows the patient to be positioned supine and induced on the flat board. The carbon frame is then placed on top of the patient (sandwiching them in position) and the arms and legs are secured with safety straps. The patient is then rotated 180 degrees and the flat board removed. The head can be secured during the rotation with either Mayfield pins, a traction device (e.g., Gardener well's tongs), or a prone pillow. ${ }^{13}$

The "sandwich and flip" rotation has several advantages. A neutral neck position can be confirmed both before and after the carbon frame is applied and the patient is sandwiched in position. This sandwiching effect means that in contrast to a MILS turn, a single individual on the flipping team who is slightly out of sync with the rest of the team will not alter the patient's neck position during the turn. It also allows prone supports to be correctly aligned for each patient before the turn, thus minimizing readjustments and pressure injuries in the prone position.

The main disadvantage of this technique is that the safe turning of the patient is a multifaceted process that requires teamwork, coordination, and acquired skill. If performed incorrectly, a "sandwich and flip" rotation can result in serious injury to the patient including the potential to fall off the table as well as injury to the personnel involved in the positioning. ${ }^{14}$ Standard of care for a "sandwich and flip," therefore, should include a "timeout" during which the equipment and the role of all personnel are double-checked prior to the maneuver. Asiedu et $\mathrm{al}^{14}$ and DiPaola et al ${ }^{15}$ propose performing a " 444 check" to ensure that the top four 


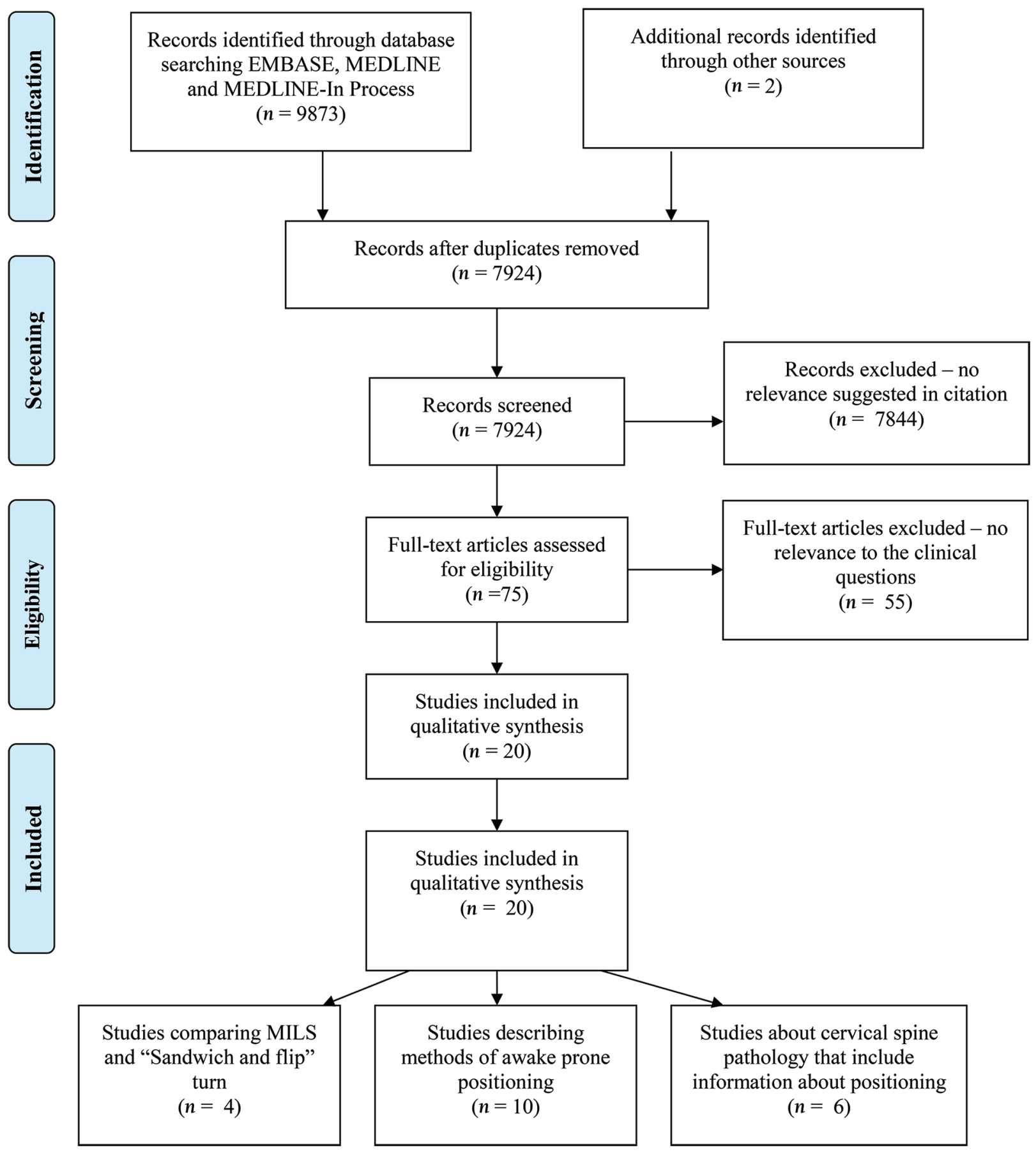

Fig. 1 Preferred Reporting Items for Systematic Reviews and Meta-Analyses (PRISMA) flow diagram.

fixation pins, the bottom four fixation pins, and the four safety belts are all secured before initiating the "sandwich and flip." Careful attention should also be paid to the monitoring cables and intravenous lines during the turn; anesthesiologists can either disconnect the lines and cables for the turn or align them so that the lines do not become entangled or disconnected during the turn. Rehearsing the maneuver with all team members improves efficiency and safety but is time-consuming.

\section{Awake prone positioning}

Two techniques of awake prone positioning have been reported in the literature. The first method involves securing the airway while the patient is awake and then the patient moves himself or herself into the prone position. ${ }^{16-18}$ The second technique involves positioning the patient prone first and then the airway is secured using either a fiber-optic intubation or a laryngeal mask airway (LMA). ${ }^{19,20}$ 
Table 1 Advantages and disadvantages of methods of prone positioning

\begin{tabular}{|c|c|c|}
\hline $\begin{array}{l}\text { Methods of prone } \\
\text { positioning }\end{array}$ & Advantages & Disadvantages \\
\hline Log roll with mils & - No additional equipment required & $\begin{array}{l}\text { - Requires coordination to ensure the spine } \\
\text { is kept inline } \\
\text { - Lack of formal teaching process } \\
\text { - Single individual mistake can result in } \\
\text { harm to patient }\end{array}$ \\
\hline $\begin{array}{l}\text { "Sandwich and } \\
\text { flip" rotation }\end{array}$ & $\begin{array}{l}\text { - Reduces neck movement } \\
\text { - Allows the prone support system to be adjusted to } \\
\text { the individual patient whilst they are in the supine } \\
\text { position-minimizing the risk of pressure injuries }\end{array}$ & $\begin{array}{l}\text { - Potential for significant patient injury if } \\
\text { performed incorrectly } \\
\text { - Learning curve involved with performing } \\
\text { the maneuver }\end{array}$ \\
\hline $\begin{array}{l}\text { Awake prone } \\
\text { positioning }\end{array}$ & $\begin{array}{l}\text { - Allows neurological assessment immediately } \\
\text { after transfer } \\
\text { - Reduces pressure injuries from poor positioning } \\
\text { - Saves on manpower }\end{array}$ & $\begin{array}{l}\text { - Requires experience and planning } \\
\text { - Lack of familiarity }\end{array}$ \\
\hline
\end{tabular}

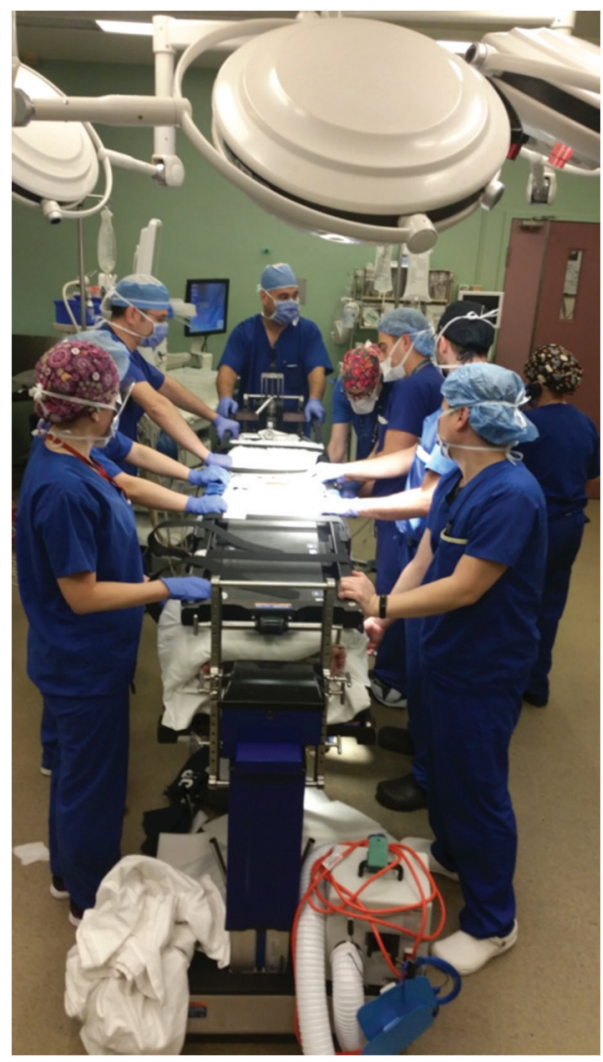

Fig. 2 Photo of a "sandwich and flip" rotation.

The literature describes many methods of securing the airway in an awake patient who is then able to position himself or herself prone. ${ }^{17,18,21-25}$ There are risks that a patient who positions themselves prone will cause further injury to the cervical spine if sedated prior to this positioning; thus, it is necessary that the patient determines the exact prone position they will move into prior to any sedation being administered. Malcharek et $\mathrm{al}^{16}$ demonstrated that this technique of patient self-positioning was successful in $78 \%$ of patients and was well tolerated by all patients with $50 \%$ of patients having no recall of the event.
The advantage of this technique is that it allows for prompt neurological assessment both after airway manipulation and prone positioning. One disadvantage is that extremely good topicalization and patient co-operation is crucial for success. Another disadvantage of awake self-positioning is that the procedure cannot be performed in all patients with cervical pathology. Patients with reduced level of consciousness or traumatic brain injury are not appropriate candidates for this method of placement. Similarly, patients with significant neurological deficit, such as quadriplegia or paraplegia, may not physically be able to move into the prone position.

Olsen et $\mathrm{a}^{19}$ have described the technique of securing the airway after the patient has moved into the prone position. After preoxygenation, general anesthesia was induced in the prone position and the airway was secured using a Proseal LMA (Intavent Orthofix; Maidenhead, United Kingdom). A trolley was placed next to the operating table until the airway was secured in case difficulties arose and the patient had to be moved back into the supine position. Authors claim that successful placement of LMA was possible in 62 out of 64 patients with no associated respiratory distress, laryngospasm, or aspiration. In addition, authors have demonstrated that this technique was faster than intubating a supine patient and then turning them prone. ${ }^{19}$ Securing the airway once the patient is in the prone position is technically more difficult; furthermore, bag mask ventilation can be very challenging in prone position. These difficulties increase the potential likelihood of running into a situation where the patient is unable to be ventilated and oxygenated, thus requiring the patient to be moved back into the supine position to place the airway. Of note, Olsen et al did not find this to be an issue during their trial. However, it may be prudent to avoid this technique in patients with an unstable cervical spine as they are known to be more difficult intubations and emergent flipping back to the supine position and intubating could place these patients at risk of a number of complications, including secondary neurological injury. 

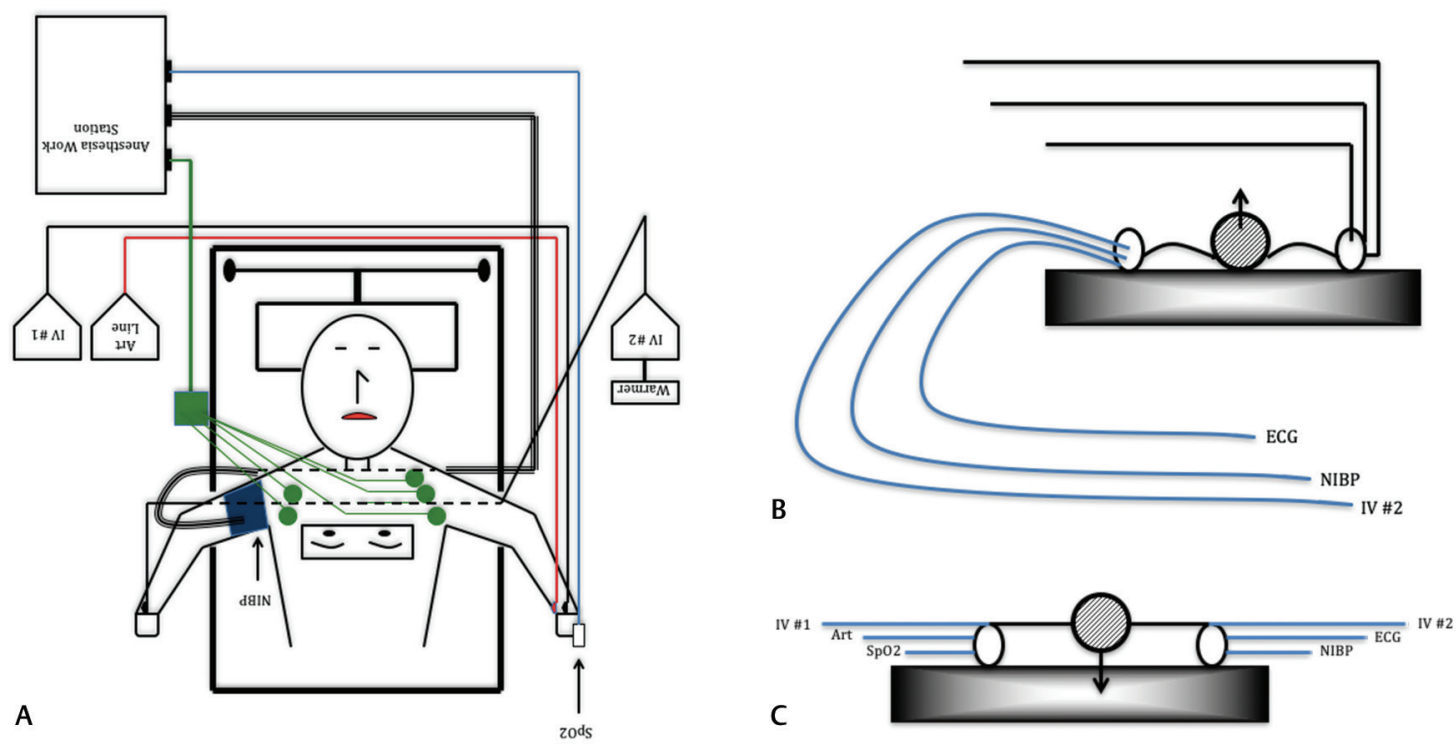

Fig. 3 Schematics of a "sandwich and flip" rotation. (A) Schematic detailing the placement of monitoring and intravenous lines to prevent entanglement or disconnection during a "sandwich and flip" rotation. The patient is set up to be turned 180 degrees to the right when moving prone. The image is a bird's eye view taken from above the patient's head. Dotted lines indicate that the monitoring or intravenous line travels underneath the table before connecting to the patient. Art line, arterial line; ECG electrodes, electrocardiogram electrodes; IV \#1, intravenous line 1; IV \#2, intravenous line 2; NIBP, noninvasive blood pressure; SpO2, saturation probe. (B) Schematic demonstrating a horizontal view of the monitoring and intravenous line placement in the same patient when supine and the movement of these lines during the "sandwich and flip" turn. Of note, the intravenous line 2, electrocardiogram lead, and the noninvasive blood pressure cuff lead pass under the patient during the turn. The circle in the middle of the diagram represents the patient's face with the arrow indicating the direction the patient is facing. (C) Schematic demonstrating the final position of the patient in the prone position with monitoring attached.

\section{Cervical spine movement with different methods of prone positioning}

Our literature review revealed four cadaveric studies comparing the degree of neck movement during a logroll using MILS and a "sandwich and flip" technique using a Jackson table. . $5,26-28^{2}$ A total of 14 cadavers were tested: 10 had C5-6 instability and 4 had C1-2 instability. An electromagnetic motion analysis device was used to measure neck movement ( - Table 2 ).

All four cadaveric studies showed reduced degree of both flexion-extension and axial-lateral rotation by over $50 \%$ with "sandwich and flip" rotation when compared to logroll with MILS. Two studies explored the type of headrest used when the patient is in the prone position after having undergone a "sandwich and flip." Of note, the type of headrest had less impact on neck instability in patients with C1-2 instability compared to those with C5-6 instability. ${ }^{15,26}$ However, in patients with C5-6 instability, using a blue foam pillow or prone view headrest provided better stabilization than Mayfield pins in terms of mediolateral and anteroposterior movement, but there was no difference in stabilization between the three methods in patients undergoing a "sandwich and flip" with C1-2 instability. 15,26

Patients with unstable cervical pathology, especially from trauma, often present to the operating theater with cervical collars or halo orthoses. DiPaola et $\mathrm{a}^{15}$ demonstrated that in these patients keeping the collar on during prone positioning reduced the amount of flexion/extension, axial rotation, and lateral bend whether the patient was turned using MILS or a "sandwich and flip" on a Jackson table. Similarly, halo orthoses should be kept on during prone positioning using the log roll technique. While halo orthoses reduce the degree of movement of the cervical spine, they do not eliminate movement completely, and significant degrees of motion can still be generated during a MILS turn. ${ }^{29}$

Table 2 Summary of cadaveric studies comparing MILS versus "sandwich and flip" rotation using a Jackson table

\begin{tabular}{|c|c|c|c|c|c|c|}
\hline Author & Date & $\begin{array}{l}\text { Number of } \\
\text { cadavers }\end{array}$ & $\begin{array}{l}\text { Cervical spine } \\
\text { pathology }\end{array}$ & $\begin{array}{l}\text { Amount of axial } \\
\text { rotation" (degrees) }\end{array}$ & $\begin{array}{l}\text { Amount of flexion/ } \\
\text { extension" (degrees) }\end{array}$ & $\begin{array}{l}\text { Amount of lateral } \\
\text { bend" (degrees) }\end{array}$ \\
\hline DiPaola et al $\left.\right|^{15}$ & 2008 & 4 & C5-6 instability & $\begin{array}{l}\text { Not available } \\
(p=0.039)\end{array}$ & $\begin{array}{l}\text { Not available } \\
(p=0.029)\end{array}$ & $\begin{array}{l}\text { Not available } \\
(p=0.019)\end{array}$ \\
\hline Dipaola et $a^{26}$ & 2009 & 4 & C1-2 instability & $\begin{array}{l}16.1 \text { vs. } 4.7 \\
(p<0.031)\end{array}$ & $\begin{array}{l}12 \text { vs. } 5 \\
(p<0.025)\end{array}$ & $\begin{array}{l}10.6 \text { vs. } 2.9 \\
(p<0.034)\end{array}$ \\
\hline Bearden et al ${ }^{27}$ & 2011 & 1 & C5-6 instability & $\begin{array}{l}29 \text { vs. } 7 \\
(p<0.001)\end{array}$ & $\begin{array}{l}31 \text { vs. } 8 \\
(p<0.001)\end{array}$ & $\begin{array}{l}20 \text { vs. } 4 \\
(p<0.001)\end{array}$ \\
\hline Prasarn et $a^{28}$ & 2012 & 5 & C5-6 instability & $\begin{array}{l}6 \text { vs. } 2.9 \\
(p<0.010)\end{array}$ & $\begin{array}{l}14.5 \text { vs. } 5.3 \\
(p<0.008)\end{array}$ & $\begin{array}{l}9.7 \text { vs. } 4.1 \\
(p<0.015)\end{array}$ \\
\hline
\end{tabular}

"MILS vs. "sandwich and flip" rotation. 


\section{Discussion}

The purpose of this review was to explore the safest method of prone positioning in patients with unstable cervical pathology. Three main methods of prone positioning are described in the literature and a "sandwich and flip" rotation is associated with less neck movement than logroll with MILS. ${ }^{15,26-28}$ For cooperative patients who are physically capable, awake self-positioning is a good alternative.

Minimizing neck movement when positioning patients prone with unstable cervical pathology reduces the risk of secondary neurological injury. One of the difficulties encountered in research in this area is that the amount of neck movement that can be tolerated without causing spinal cord compression is unclear and appears to vary with the underlying pathology and individual patient. From clinical experience, a patient who develops radiculopathic symptoms on minimal neck rotation seems to have less toleration of neck movement than a patient with no symptoms during normal cervical spine range of motion. The prone position worsens canal stenosis in patients with cervical myelopathy compared to supine positioning, reducing the margin for error. ${ }^{7}$ Second, in the prone position, vena caval compression can lead to a reduction in spinal cord blood flow, thus further reducing the amount of spinal cord compression tolerated. ${ }^{7}$ The theoretically increased risk of neurological injury in patients undergoing prone positioning is supported in clinical practice. Kutteruf et $\mathrm{al}^{6}$ found on a review of closed claims cases that permanent disabling injuries were more likely to occur in patients undergoing spine surgery in the prone position (odds ratio, 3.50; $p=0.013$ ).

In general, all anesthetic agents act on the spinal cord to produce immobility in a dose-dependent manner. ${ }^{30-32}$ In patients undergoing general anesthesia, motor evoked potential (MEP) and somatosensory evoked potential (SSEP) monitoring before and after positioning can be useful, especially in patients who are at high risk of secondary injury. ${ }^{33}$ SSEPs monitor the ascending sensory tracts in the posterior columns, while MEPs assess the descending motor pathways in the anterior and posterolateral corticospinal tracts. ${ }^{34}$ Baseline recordings are taken at the start of the procedure and reduction in amplitude of the SSEP recordings or an increase in the voltage required to stimulate an MEP response suggests an acute spinal cord injury. ${ }^{34}$ Fehlings et a ${ }^{35}$ published a systematic review that concluded that SSEP and MEP monitoring during spine surgery was a sensitive and specific way of monitoring spinal cord function. Furthermore, they found that there was low level evidence that appropriately responding to a neuromonitoring change reduced the rate of perioperative neurological deterioration.

Awake, cognitively intact patients with an unstable cervical spine can, however, still develop spinal cord compression during positioning. Deem et $\mathrm{al}^{36}$ describe the case of a 60-year-old man with severe cervical stenosis undergoing thoracolumbar surgery in the prone position. He underwent awake fiber-optic intubation and careful positioning, and had a normal neurological assessment after moving into the prone position. On emerging from anesthesia, however, it became clear he had developed cervical central cord syndrome. The authors acknowledge that despite careful positioning, subtle amounts of positioning-related injury might have still occurred, which could have contributed to the patient's secondary neurological injury.

Irrespective of the technique used to achieve prone positioning, the institutional practice and surgeon's preference vary. All methods of prone positioning patients with unstable cervical spines require careful planning and execution. Individual institutions may have developed their own policies, procedures, and training for particular methods of positioning these patients, and, in the best interests of patients, it may not be appropriate to change an institution's carefully implemented practices. If a change in the method of prone positioning is planned, then educating all members of staff who will be involved in the new procedure is crucial for success.

Our review of the literature does have many limitations. In the cadaveric studies, the models studied have altered tissue architecture, which may change the degree of cervical spine movement compared to that of live patients. Hence, the benefit of a "sandwich and flip" technique may not be completely applicable to clinical practice. Similarly, awake prone positioning, while shown to be a highly successful technique, may be subject to publication bias. ${ }^{16,19}$ Case reports about failed attempts at awake prone positioning are unlikely to be written or published. Moreover, studies on awake self-positioning are likely to be conducted in centers accustomed to the complications of performing the procedure and may, therefore, have higher success rates than centers first implementing this technique.

\section{Conclusion}

With the limited evidence available, our review suggests that a "sandwich and flip" is the safest way to turn a patient with cervical pathology into a prone position for surgery. Awake self-positioning of a patient is a good alternative that allows for rapid neurological assessment post-intubation and for repositioning.

\section{Conflict of Interest}

None declared.

\section{References}

1 Nguyen HV, Ludwig S, Gelb D. Osteoporotic vertebral burst fractures with neurologic compromise. J Spinal Disord Tech 2003;16(1):10-19

2 Colterjohn NR, Bednar DA. Identifiable risk factors for secondary neurologic deterioration in the cervical spine-injured patient. Spine 1995;20(21):2293-2297

3 Harrop JS, Sharan AD, Vaccaro AR, Przybylski GJ. The cause of neurologic deterioration after acute cervical spinal cord injury. Spine 2001;26(4):340-346

4 Yablon IG, Ordia J, Mortara R, Reed J, Spatz E. Acute ascending myelopathy of the spine. Spine 1989;14(10):1084-1089

5 Farmer J, Vaccaro A, Albert TJ, Malone S, Balderston RA, Cotler JM. Neurologic deterioration after cervical spinal cord injury. J Spinal Disord 1998;11(3):192-196 
6 Kutteruf R, Wells D, Stephens L, Posner KL, Lee LA, Domino KB. Injury and liability associated with spine surgery. J Neurosurg Anesthesiol 2018;30(2):156-162

7 Crosby ET. Airway management in adults after cervical spine trauma. Anesthesiology 2006;104(6):1293-1318

8 Crosby ET, Lui A. The adult cervical spine: implications for airway management. Can J Anaesth 1990;37(1):77-93

9 Diedrich DA, Rose PS, Brown DR. Airway management in cervical spine injury. Curr Anesthesiol Rep 2013;3(3):197-204

10 Brimacombe J, Keller C, Künzel KH, Gaber O, Boehler M, Pühringer F. Cervical spine motion during airway management: a cinefluoroscopic study of the posteriorly destabilized third cervical vertebrae in human cadavers. Anesth Analg 2000;91(5):1274-1278

11 Hirabayashi Y, Fujita A, Seo N, Sugimoto H. Cervical spine movement during laryngoscopy using the Airway Scope compared with the Macintosh laryngoscope. Anaesthesia 2007;62(10):1050-1055

12 Conrad BP, Rossi GD, Horodyski MB, Prasarn ML, Alemi Y, Rechtine GR. Eliminating log rolling as a spine trauma order. Surg Neurol Int 2012;3(Suppl 3):S188-S197

13 Vanderhave KL, Chiravuri S, Caird MS, et al. Cervical spine trauma in children and adults: perioperative considerations. J Am Acad Orthop Surg 2011;19(6):319-327

14 Asiedu GB, Lowndes BR, Huddleston PM, Hallbeck S. "The Jackson Table Is a Pain in the...": A Qualitative Study of Providers' Perception Toward a Spinal Surgery Table. J Patient Saf 2016;14(1):21-26

15 DiPaola MJ, DiPaola CP, Conrad BP, et al. Cervical spine motion in manual versus Jackson table turning methods in a cadaveric global instability model. J Spinal Disord Tech 2008;21(4):273-280

16 Malcharek MJ, Rogos B, Watzlawek S, et al. Awake fiberoptic intubation and self-positioning in patients at risk of secondary cervical injury: a pilot study. J Neurosurg Anesthesiol 2012;24(3):217-221

17 Avitsian R, Lin J, Lotto M, Ebrahim Z. Dexmedetomidine and awake fiberoptic intubation for possible cervical spine myelopathy: a clinical series. J Neurosurg Anesthesiol 2005;17(2):97-99

18 Douglass J, Fraser J, Andrzejowski J. Awake intubation and awake prone positioning of a morbidly obese patient for lumbar spine surgery. Anaesthesia 2014;69(2):166-169

19 Olsen KS, Petersen JT, Pedersen NA, Rovsing L. Self-positioning followed by induction of anaesthesia and insertion of a laryngeal mask airway versus endotracheal intubation and subsequent positioning for spinal surgery in the prone position: a randomised clinical trial. Eur J Anaesthesiol 2014;31(5):259-265

20 Sriganesh K, Ramesh VJ, Veena S, Chandramouli BA. Dexmedetomidine for awake fibreoptic intubation and awake self-positioning in a patient with a critically located cervical lesion for surgical removal of infra-tentorial tumour. Anaesthesia 2010;65(9):949-951

21 Song J, Shin YS, Shim YH. The effect site concentration of remifentanil for suppressing severe cough and defensive movement during awake fibreoptic nasotracheal intubation in patients undergoing cervical spine surgery: 9AP1-3. Eur J Anaesthesiol 2011;28:128
22 Ehsan AQ Obaid AS, Hussain AS, Nesar AM. Awake Airtraq intubation in an immobile neck-a case report. Acta Anaesthesiol Belg 2012;63(3):135-137

23 Sener EB, Sarihasan B, Ustun E, Kocamanoglu S, Kelsaka E, Tur A. Awake tracheal intubation through the intubating laryngeal mask airway in a patient with halo traction. Can J Anaesth 2002;49(6):610-613

24 Mahmoudpour A, Hoseinzadeh H, Far AP, Sanaie S. Awake tracheal intubation via intubating laryngeal mask versus direct laryngoscopy and cervical spine excursion. Pak J Med Sci 2007;23(2):238-241

25 Wong JK, Tongier WK, Armbruster SC, White PF. Use of the intubating laryngeal mask airway to facilitate awake orotracheal intubation in patients with cervical spine disorders. J Clin Anesth 1999;11(4):346-348

26 Dipaola CP, Conrad BP, Horodyski M, Dipaola MJ, Sawers A, Rechtine GR II. Cervical spine motion generated with manual versus Jackson table turning methods in a cadaveric c1-c2 global instability model. Spine 2009;34(26):2912-2918

27 Bearden BG, Conrad BP, Horodyski M, Rechtine GR. Motion in the unstable cervical spine: comparison of manual turning and use of the Jackson table in prone positioning. J Neurosurg Spine 2007;7(2):161-164

28 Prasarn ML, Horodyski M, Dubose D, et al. Total motion generated in the unstable cervical spine during management of the typical trauma patient: a comparison of methods in a cadaver model. Spine 2012;37(11):937-942

29 DiPaola CP, Sawers A, Conrad BP, et al. Comparing cervical spine motion with different halo devices in a cadaveric cervical instability model. Spine 2009;34(2):149-155

30 Eger EI II, Raines DE, Shafer SL, Hemmings HC Jr, Sonner JM. Is a new paradigm needed to explain how inhaled anesthetics produce immobility? Anesth Analg 2008;107(3):832-848

31 Hendrickx JF, Eger EI II, Sonner JM, Shafer SL. Is synergy the rule? A review of anesthetic interactions producing hypnosis and immobility. Anesth Analg 2008;107(2):494-506

32 Kungys G, Kim J, Jinks SL, Atherley RJ, Antognini JF. Propofol produces immobility via action in the ventral horn of the spinal cord by a GABAergic mechanism. Anesth Analg 2009;108(5):1531-1537

33 Stevens, RD, et al. Critical care and perioperative management in traumatic spinal cord injury. J Neurosurg Anesthesiol 2003;15(3): 215-229

34 Pajewski TN, Arlet V, Phillips LH. Current approach on spinal cord monitoring: the point of view of the neurologist, the anesthesiologist and the spine surgeon. Eur Spine J 2007;16Suppl 2 :S115-S129

35 Fehlings MG, Brodke DS, Norvell DC, DettoriJR. The evidence for intraoperative neurophysiological monitoring in spine surgery: does it make a difference? Spine 2010;35(9 Suppl):S37-S46

36 Deem S, Shapiro HM, Marshall LF. Quadriplegia in a patient with cervical spondylosis after thoracolumbar surgery in the prone position. Anesthesiology 1991;75(3):527-528 